\title{
An Improved Local Equilibrium Contrast Enhancement Algorithm for Infrared Laser Images
}

\author{
Yuhong Li, Jianzhong Zhou, Wei Ding, Shan Ding \\ Digital Engineering and Simulation Centre \\ Huazhong University of Science and Technology \\ Wuhan 430074, China \\ liyuhong@hust.edu.cn
}

\begin{abstract}
An improved local equilibrium contrast enhancement algorithm based self-adaptive contrast enhancement algorithm is proposed for infrared laser images, in which the image pixel value histogram is divided into three parts: background and noise area, targets area, and uninterested area. The targets parts are highlighted, while the background and noise parts and the uninterested parts are restrained. A comprehensive qualitative and quantitative image enhancement performance evaluation is presented to verify the proposed theory and algorithm validity, efficiency and reasonability. The experimental results indicate that the proposed algorithm can greatly improve the global and local contrast for both near infrared images and far infrared laser images while efficiently reducing noise in the infrared laser images, and the visual quality of enhanced image is obviously better than the enhancement of the traditional histogram equalization, double plateaus histogram equalization algorithm, etc.
\end{abstract}

Index Terms-Infrared Laser Images; Grey Transformation; Evaluation of Image Quality; Selfadaptive Threshold;

\section{INTRODUCTION}

Today, infrared laser techniques are applied widely in many domains, such as military field, laser-guided tracking, laser radar, laser targeting, and others. The current applications on laser measurement are included in unit array detection and spot imaging two main fields. To reduce the cost, a CCD camera and an un-cooled imaging device may have to be used for near infrared laser and far infrared laser measuring respectively. Infrared laser images taken under the natural illumination conditions reveal the grey distributions information of the images, express the object and the environment temperature and radiating power. Due to the sensitivity, resolution, noise and other inherent characteristics of infrared imaging

This work was partially supported by the Grant from National Key Basic Research Program of China ( 973 Program, No.2007CB714107) and NSFC of China (No.10971225), the Scientific Research Foundation for the Returned Overseas Chinese Scholars, State Education Ministry, and the Talent Recruitment Foundation of HUST. devices, infrared laser images commonly have some shortcomings such as high background, low contrast, fuzzy edge of the target, low image signal to noise ratio (SNR) and so on. Especially, the noise would deteriorate the image quality. Most of infrared laser images always have very little priori-information and smooth boundaries or even with discontinuous boundaries. In particular for the infrared small dim target image, the targets and the details are drowned in the large background context, which brings great difficulties to the image analysis, calculation, identification and applications. It is very important to enhance the contrast the infrared laser images, which will affect the quality of segmentation and laser energy calculation. Algorithms, which are used widely to enhance the contrast for infrared image, are histogram equalization, histogram matching, double plateaus histogram equalization, gray level transform and un-sharp mask algorithm [1].

Histogram equalization is a popular enhancement algorithm in visible light images, which mainly performs the grey level adjustment according to the accumulating histogram to stretch the dynamical range and enhances the contrast as a consequence. Now, it is well known that histogram equalization enhancement is not applicable for the infrared images enhancement ${ }^{[1,2,5,6,10]}$. As a modification of histogram equalization, double plateaus histogram equalization could constrain the background and noises by setting a higher threshold value $T_{1}$ as the upper limit, and could magnify the dim small targets and the details of the image by setting a lower threshold value $^{T_{2}}$ as the lower limit $\left(T_{1} \geq T_{2}\right)$. Though the double plateaus histogram equalization algorithm can be found in practice and applications, some shortcoming with its applications are still unresolved. Firstly, it is very difficult to determine the platform thresholds. Song and his collaborators presented in [2] a strategy with 20\%-30\% and $5 \%-10 \%$ of the total pixels as the upper limit threshold and the lower limit threshold respectively. However, the strategy could not be applied for all images. In particular, it is not good for the infrared laser images enhancement. To overcome this shortcoming, we normally keep trying to adjust the upper limit threshold and lower limit threshold by observe the change of histogram to achieve the better enhancement results. Secondly, the algorithm only moderately restrains the 
background and noise, in which it only restrains those parts with grey level value greater than upper limit threshold $T_{1}$. In that way, the best restrained effects for the background and noise cannot be achieved. In order to restrain the background and noise to a large extent, the value of the upper limit threshold $T_{1}$ will be chosen very small, so that the accuracy and difficulties of thresholds selection will be greatly increased. Therefore, this algorithm for enhancement of the infrared laser images with strong background and noise could be further improved.

The common disadvantage of above algorithms is that the noise in the image is magnified while the contrast is enhanced, which cause the ambition of targets, [3-7]. Enhancement of a single image ignores the additional information provided temporally. Traditional histogram equalization and gray-scale transformation algorithms can't realize the enhancement of the target in the infrared image effectively. In response to these problems, based on the piecewise linear transform image enhancement technique, an improved local equilibrium contrast enhancement algorithm will be presented to effectively restrain the background and noise, and highlight the targets in the images in this paper. The algorithm combines the environment information analysis for infrared laser image contrast enhancement. Experimental results show that the new algorithm can efficiently enhance the global contrast and the local contrast for the far infrared laser image, and the visual quality of enhanced image employing the new algorithm is obviously better than the traditional histogram equalization, double plateaus histogram equalization algorithm, etc.

The rest of this paper is organized as follows. Section 2 presents a novel self-adaptive threshold piecewise linear transformation enhancement algorithm after briefly introducing the piecewise linear transformation enhancement. Section 4 introduced the algorithm of improved local histogram equilibrium. And some experimental results and comparison are given in Section 5. Section6 draws the conclusions

\section{SELF-AdAPTIVE PieCEWISE LiNEAR TRANSFORM}

The piecewise linear transformation enhancement is somewhat similar with the linear transformation enhancement, and is different from the later one. The piecewise linear transformation is not truly linear transformation, but is linear transformation in every subsegments. That is the grey levels interval of images will be divided into two or even more segments, the linear transformation enhancement will be performed in very segments. The advantage for piecewise linear transformation enhancement is that the details of targets can be stretched, and the uninterested parts with background and noise can be relatively restrained accordance with the different applications.

$$
y=\left\{\begin{array}{lr}
y_{1} * \frac{x}{x_{1}}, & x<x_{1} \\
y_{1}+\left(y_{2}-y_{1}\right) * \frac{x-x_{1}}{x_{2}-x_{1}}, & x_{1} \leq x \leq x_{2} \\
y_{2}+\left(255-y_{2}\right) * \frac{x-x_{2}}{255-x_{2}}, & x>x_{2}
\end{array}\right.
$$

where $\left(x_{1}, y_{1}\right)$ and $\left(x_{2}, y_{2}\right)$ denote the coordinate of two turning points respectively.

As we have described above, the conventional thresholds selection are manually carried out. This interactive input method is flexible, while the best possible effects of enhancement should be reached through repetitive testing for the suitable thresholds. In order to solve this problem, a self-adaptive threshold calculation algorithm will be presented based on the statistic of the histogram and the estimate of the grey level contained in the targets areas. Threshold ${ }^{x_{1}}$ denotes the boundary between background (including noise) and targets areas; threshold $x_{2}$ denotes the boundary between targets areas and uninterested areas.

Come back to the infrared laser images analysis, if we can determine the boundaries for background, targets and uninterested areas in the image histogram, that is to determine the values of $x_{1}$ and $x_{2}$. And we call the interval $\left[0, x_{1}\right)$ the background areas, the interval $\left[x_{1}, x_{2}\right]$ the targets areas, and the interval $\left(x_{2}, 255\right]$ the uninterested areas. Then, we can apply the piece-wise linear transformation with that values of $x_{1}$ and $x_{2}$ to stretch or suppress the grey levels of the images for the purpose of restraining background and noise, and highlighting targets.

Let us first analysis some characteristics of infrared laser images. The below Figure 1 are infrared laser images taken by a CCD camera and a LWTIFPA camera respectively in our designed infrared laser system with their histogram, [8]. The peak values in those histograms correspond to the majority grey level in background and noise. So, in order to restrain the background and noise, it is better to choose the threshold $x_{1}$ is close to the grey level value of the peak value. Then, when we suppress the interval $\left[0, x_{1}\right]$, the corresponding interval in $y$-axis including the peak of the histogram will be suppressed, which just contain the background and noise parts in the image. In response to these characteristics of the peak value of the histogram, we should first count the statistic histogram of the original image, and then compare every grey level value probabilities to get the grey level value corresponding to the histogram peak value. If the above grey level value was selected as the threshold ${ }^{X_{1}}$, then the left hand side of the peak value will be restrained, and the right hand side of the peak value will be enhanced. In order to restrain most of the background and noise, the threshold $x_{1}$ should be theoretically chosen a littler bit bigger than the grey level value corresponding to the peak value in the histogram. However, the peak strip is a 
little narrow in the histogram of infrared images (both for near or far infrared laser images), a small adjustment can be carried out to get the threshold ${ }^{x_{1}}$, i.e.

$x_{1}=$ the grey level value corresponding to the peak value $+n$

where $n$ is a integer, for convenient we can set it to take value from ${ }^{[0,10]}$. We take an adjustable parameter $n$ to guarantee that the right hand side of the peak in the histogram can be restrained somehow. However, the experimental results show us that the value of $n$ ( $0 \leq n \leq 10$ ) will not cause the dramatic change of the enhancement of images. So, we leave too much analysis and further research out here.

Since there is no information on the scope of the grey level values of targets in the histogram, we cannot get the threshold $x_{2}$ from the histogram directly. To solve this problem, the grey level values of targets should be estimated. From the previous analysis, we can see that the threshold $x_{1}$ denotes the demarcation point between background and targets, which means that the threshold $x_{1}$ is the lower limit of grey level values of the targets in the image; and the threshold $x_{2}$ denotes the demarcation point between targets and the uninterested areas, which means that the threshold $x_{2}$ is the upper limit of grey level values of the targets in the image. Hence, the threshold $x_{2}$ can be got through estimating the maximum grey level value on the targets. The scheme is as following:

1. Choose one point in the target area randomly;

2. Calculate the median grey value around the chosen point, normally taking the cross-window centered the chosen point (vertical and horizontal length of the cross denote by $\mathrm{N}, \mathrm{N}=3,5,7 \ldots$;
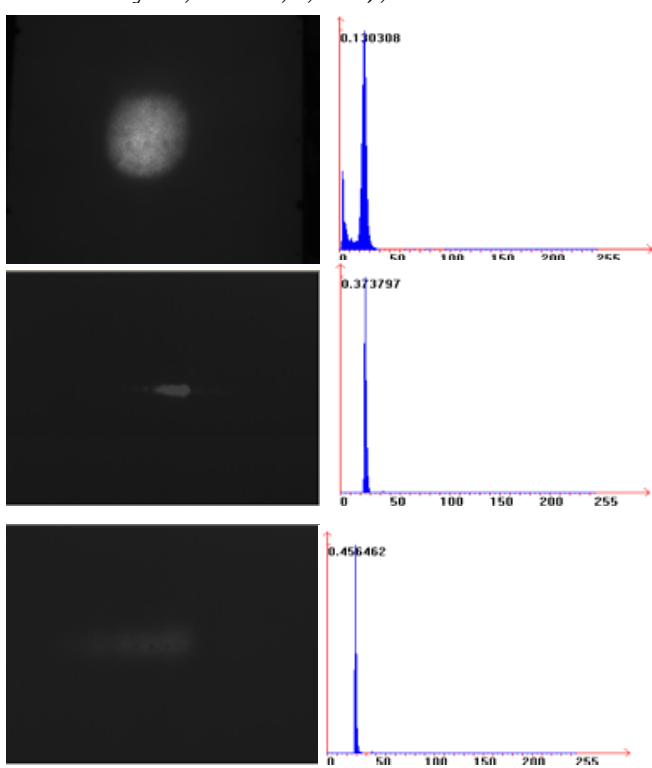

Figure 1. A Near and A Far Infrared Laser Image with their histogram

3. Denoting the chosen point by o, letting $N=5$, taking $5 \times 5$ cross window as the following Figure 4,

$M=\frac{1}{9}(f(o)+f(a)+f(b)+f(c)+f(d)+f(e)+f(f)+f(g)+f(h))$ where, $f(t)$ denotes the grey level value of point $t, t=0$, a, b, c, d, e, f, g, h.

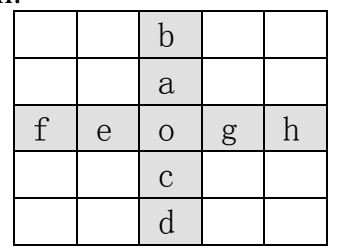

Figure 1. A $5 \times 5$ Cross Window

4. Repeat step 1 and step $2 \mathrm{M}$ times, then we get $\mathrm{M}$ median grey level values.

5. Take the maximum of the $\mathrm{M}$ median grey level values as the threshold ${ }^{X_{2}}$.

Since there is some uncertainty when we choose the target point, we should repeat step 3 and 4 to avoid the perturbation of uncertainty and to increase the accuracy of the estimate of the threshold ${ }^{X_{2}}$.

In summary, we have got the threshold $x_{1}$ by the statistic and analysis the histogram, and the threshold $x_{2}$ by the grey level value estimate of the target areas. In this way, the thresholds are calculated via the adaptive algorithm considering the influence of background and noise on the targets. Comparing with the conventional piecewise linear transformation algorithm, the uncertainty is effectively decreased, and the human computer interactive calculation complexity is reduced in this adaptive algorithm.

\section{IMPROVED LOCAL HISTOGRAM EQUILIBRIUM}

It is put into effect that the infrared image is improved to a great extent by adaptive linear transformation expressed above, which is simple and fast, and suitable for the processing of real-time infrared. However, it is worthwhile to note that the threshold $x_{2}$ obtained by interactive processing has features of randomness and blindness to a certain degree. In the experiment, we found that there exist certain changes of $x_{2}$, when we chose different targets manually. It will lead to certain disparity on the enhancement effects of spot image in every experiment, thus it is unable to reach ideal optimal effect through choosing point by once interactive take points.

The reason for the changes of $x_{2}$ have great influence on the enhancement results is that it didn't change the relative size and position of grayscale among gray segment, which result in that the chosen value of the endpoint value in gray segment plays an important part on the result of the experiment.

Of course, in the actual application process, we hope we could achieve the best effect through once interactive take points, and at least the results should be vary not so significant. This needs some methods which can reduce the impact of the randomness, and the domination of $x_{2}$ on the result of the experiments.

The background and noise are compressed into the position of gray 0 in the algorithm introduce in chapter 3 , and the gray range uninterested is compressed into the 
position of gray 255, then [1,254] will be the range after the stretch of target gray. However, the stretch is linear which didn't change the relative size and position of the gray level within the target segment. From a certain angle, the result of this procession remains space and necessary to be processed. In this paper, we take the way of improved histogram equilibrium for further processing. But, the balanced object is not the whole image processed by algorithm in 3, but just the gray segment [1,254] after the target tensile. In order to distinguish from ordinary histogram equalization, we call it local histogram equilibrium, and the principle of both of them is the same, therefore, local histogram equilibrium has the disadvantages which ordinary one has[11]: (1)it is difficult to reach the maximum gray range allowed by the image formats for the actual gray-scale range of the output image;(2)although the gray distribution histogram of the output image is close to evenly distributed, there maybe exists some differences between the value and the ideal one, and it is not the optimal value.(3)the gray level of the output image may be excessively merged, and since the swallow of the gray, it is easy to lose the information of the image. Based on these shortcomings, an improved histogram equilibrium enhancement algorithm is introduced in literature[11]. It will be used in this paper and will be mapped into local gray segment to realize further improvement. The idea of the algorithm will be introduced as follows:

The basic idea of improved histogram equilibrium is that: fixing the position of the gray level $i$, which refers to the gray histogram of the original image, according to the ratio of the probability accumulative by the left and right sides. Suppose $\mathrm{j}$ is the corresponding level after take histogram equalization on the gray level i. so:

$$
\begin{gathered}
j:(n-1-j)=\left(\sum_{k=0}^{i-1} p_{k}\right):\left(\sum_{k=i^{+}}^{n-1} p_{k}\right) \\
j=(n-1) \frac{\sum_{k=0}^{i=1} p_{k}}{\sum_{k=0}^{i * 1} p_{k}+\sum_{k=i_{1}}^{n \cdot 1} p_{k}} \\
j=(n-1) \frac{\sum_{k=0}^{i-1} p_{k}}{1-p_{i}}=(n-1) \frac{\sum_{k=0}^{i-1} q_{k}}{Q-q_{i}}
\end{gathered}
$$

Where, $\mathrm{n}$ is the gray level of the image, $\mathrm{Q}$ is the total pixel number, $p_{k}$ is the statistics probability of gray level k, $q_{k}$ is the pixel number of gray level k.

We apply the idea introduced above to a gray segment, due to the know gray segment [1,254] after target tensile, so that actually it is used to aim at the gray segment $[1,254]$. Therefore, we could get the final result after we made corresponding adjustment of the gray level $\mathrm{j}$ get from histogram equilibrium on the gray level $\mathrm{i}$ in the gray segment, it is expressed as follows:

$$
j=(n-1-1) \frac{\sum_{k=1}^{i-1} p_{k}^{\prime}}{1-p_{i}^{\prime}}=(n-1-1) \frac{\sum_{k=1}^{i-1} q_{k}}{Q^{\prime}-q_{i}}
$$

Where, ${ }^{p_{i}^{\prime}}$ represents probability of the gray level i correspond with gray segment $[1,254], Q^{\prime}$ refers to the total number of pixel in gray segment [1,254].

$$
p_{i}^{\prime}=\frac{p_{i}}{1-p_{0}-p_{255}}
$$

After the using of the local histagram equilibrium expressed above, the final enhanced results rely a lot on the gray distribution in the gray segment, which greatly weakened the decesive role of the gray segment endpoints. We can get obviously from the results of the experiments that although peole get different value of $x_{2}$ every time, the results obtained are almost the same, which solved the problems we face. On the other hand, it just estabished linear tensile on the target gray segment of linear transformation, which din't change its internal components, after the application of local histagram equalization, it changed the gray distribution in the gray segment, which improved the overall enhancement effect a lot.

\section{CRETERIA OF IMAge QuALiTy}

Employing the measure function proposed in [9-10], a criterion for evaluating the quality for enhanced image is proposed to compare the effects of different enhancement algorithms below. But it should be mentioned here, the image quality evaluation cannot be relied only on those calculation results. Since they only reflect the image quality in some extents, but not in whole, the final image quality evaluation criteria still have to rely on people's subjective perception. The following formulae are applied to calculate the quality of the enhanced images:

$$
C_{\text {contrast }}=\frac{1}{M N} \sum_{x=0}^{M-1} \sum_{y=0}^{N-1} g^{2}(x, y)-\left|\frac{1}{M N} \sum_{x=0}^{M-1} \sum_{y=0}^{N-1} g(x, y)\right|^{2}
$$

$S N R=10 \lg \left\{\frac{\frac{1}{M N} \sum_{x=0}^{M-1} \sum_{y=0}^{N-1} f(x, y)^{2}}{\frac{1}{M N} \sum_{x=0}^{M-1} \sum_{y=0}^{N-1}[g(x, y)-f(x, y)]^{2}}\right\}$,

$C_{\text {total }}=\frac{C_{\text {contrast }} * S N R}{\beta}$,

$M S E=\frac{\sum_{x=0}^{M} \sum_{y=0}^{N}[g(x, y)-f(x, y)]^{2}}{(L-1)^{2}}$,

where $\mathrm{M}, \mathrm{N}$ denotes the width and height of the enhanced image respectively, $\mathrm{L}$ denotes the rank of the grey level 
of the image, $\beta$ is a constant, $f(x, y), g(x, y)$ denotes the grey level at coordinate $(x, y)$ of the original and the enhanced image respectively, $C_{\text {contrast }}$ denotes the contrast index, SNR denotes the signal to noise ratio of the enhancement of image, $C_{\text {total }}$ denotes the quality of the enhanced image, and MSE denotes the mean square error. Bigger is $C_{\text {contrast }}$, stronger is the contrast of the enhanced image. Bigger is $S N R$, more noise have been filtered through the image enhancement. We combine $C_{\text {contrast }}$ with $S N R$ in the calculation of $C_{\text {total }}$ to evaluate the visual quality of the enhanced image. Bigger is $C_{\text {contrast }}$, better is the quality of the enhanced image. Smaller is $M S E$, better is the quality of image fidelity of the enhanced image.

\section{EXPERIMENTS AND RESUlTS}

A large number of experiments have been carried out to assess the performance of the proposed contrast enhancement algorithm for the infrared (both near and far) laser measuring system. The enhancement results and their histograms for the infrared laser image in Figure 1 are shown in Figure 3 and Figure 4 respectively.
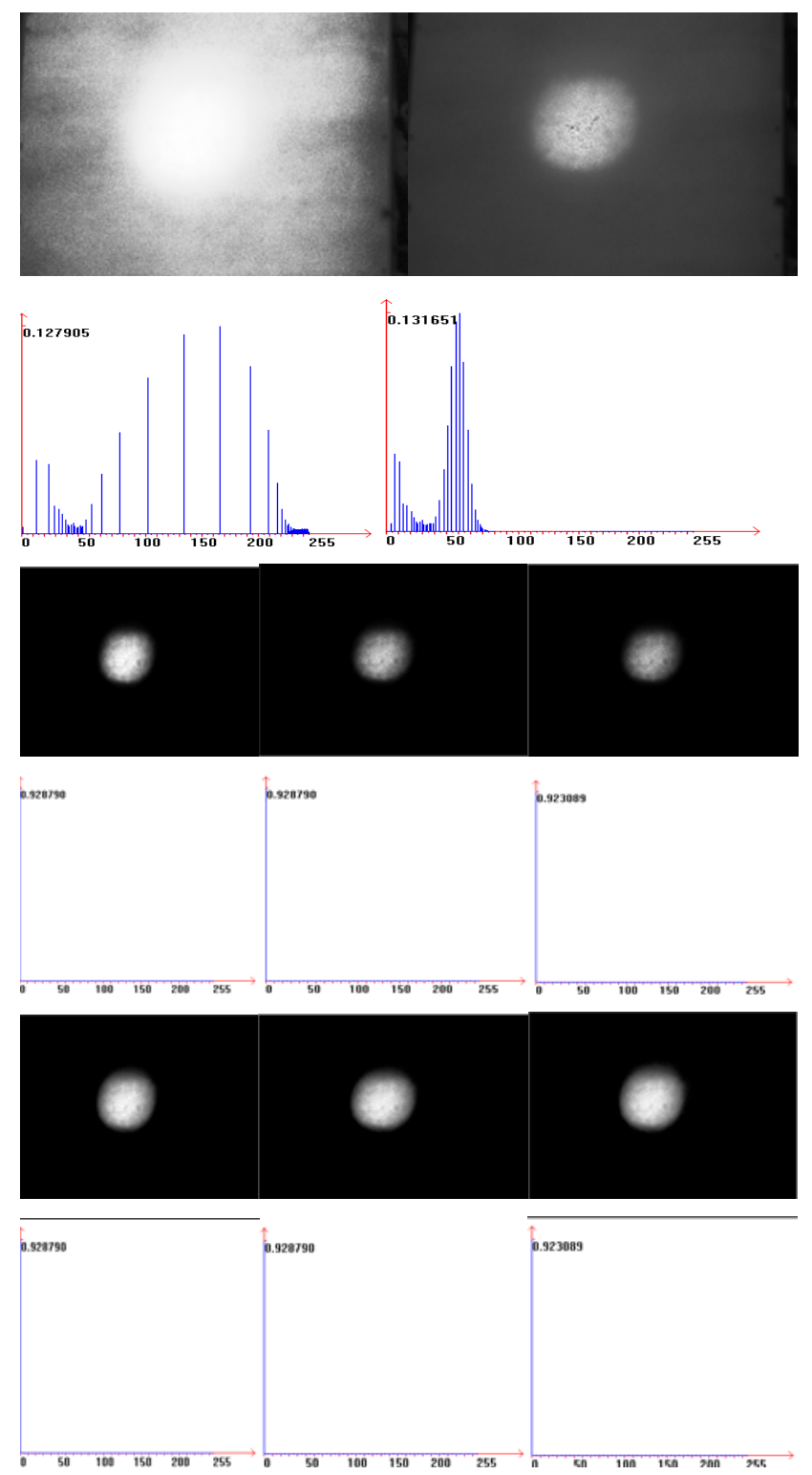

Figure 2. a)Histogram Equalization of Near Infrared Laser Image with its histogram b)Double Plateaus Histogram Equalization of Near Infrared Laser Image with its histogram (Upper Threshold $=0.013$, Lower Threshold $=0.005$ ) c) Self-adaptive Enhancement of Near Infrared Laser Image with its histogram ( $1=0, \mathrm{y} 2=240$, $\mathrm{n}=6, \mathrm{x} 1=31$,from left to right the value of $\mathrm{X} 2$ is setting as $137,153,200$ respectively) 

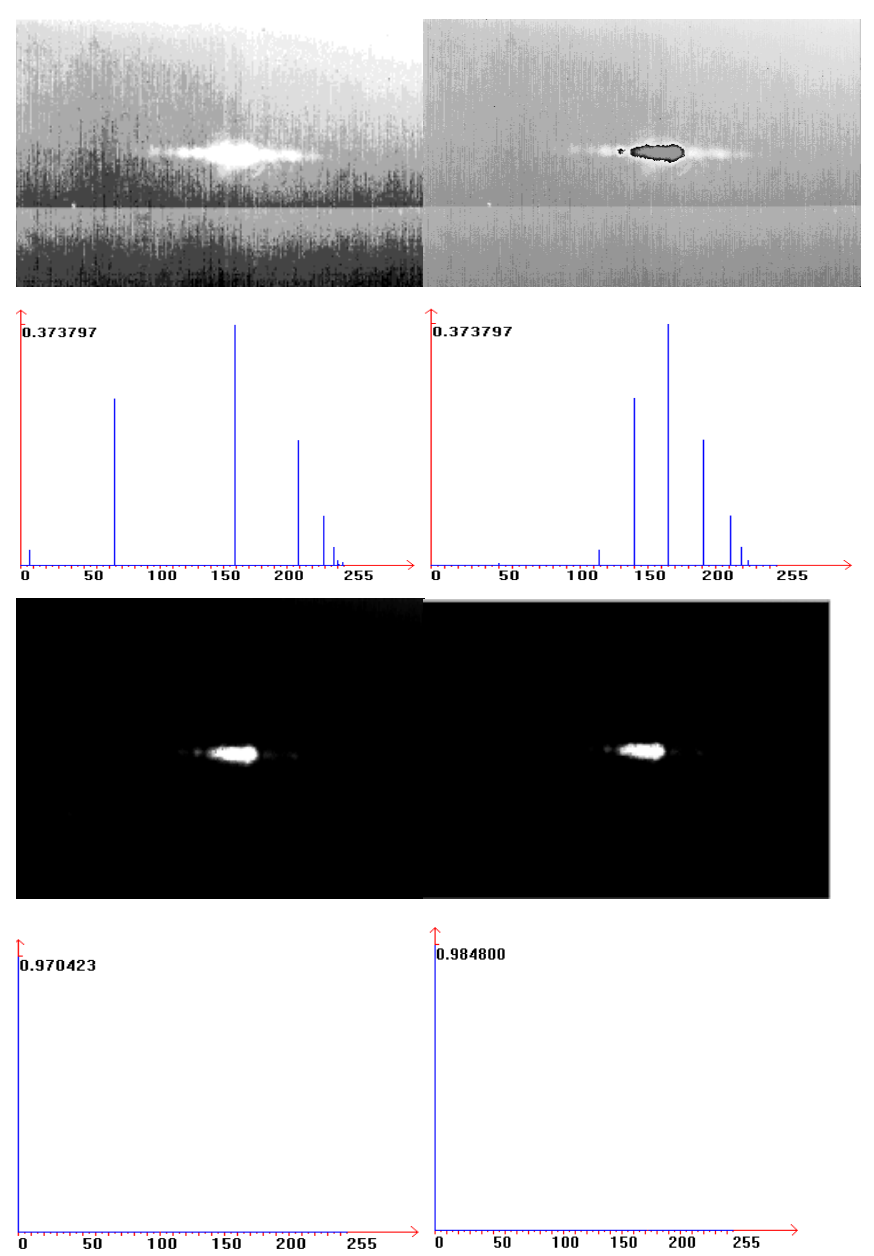

Figure 3. a) Histogram Equalization of Far Infrared Laser Image with its histogram b)Double Plateaus Histogram Equalization of Far Infrared Laser Image with its histogram (Upper Threshold=0.1, Lower Threshold=0.02) Self-adaptive Enhancement of Far Infrared Laser Image with its histogram ( $11=0, y 2=240, n=5, X 1=30 \times 2=63$ ).

In order to explain the efficiency of the self-adaptive enhancement algorithm, employing the evaluation criterion proposed in section III, the comparison of results are list respectively in Table I for the near infrared laser image and in Table II for the far infrared laser image.

From the experimental results, we can see:

1. When applying the histogram equalization on the infrared laser images, the whole image becomes brighter and the whole contrast of the image is reduced, which cause some difficulties to identify the targets in image.

2. When applying Double Plateaus Histogram equalization algorithm, there exist difficulties to select the appropriate upper limit and lower limit, which needs more trial and may cause more error. And the effects of restraining the background and noise is not quite apparent, the effects of enhancement is not well.

3. From the comparison of results list in Table I and Table II, we can see that the proposed self-adaptive enhancement algorithm reach the biggest $C_{\text {total }}$, and smallest $M S E$, which reflect that the proposed algorithm is better both for near infrared laser image and for far infrared laser image in some extents than the traditional histogram equalization and double plateaus histogram equalization.
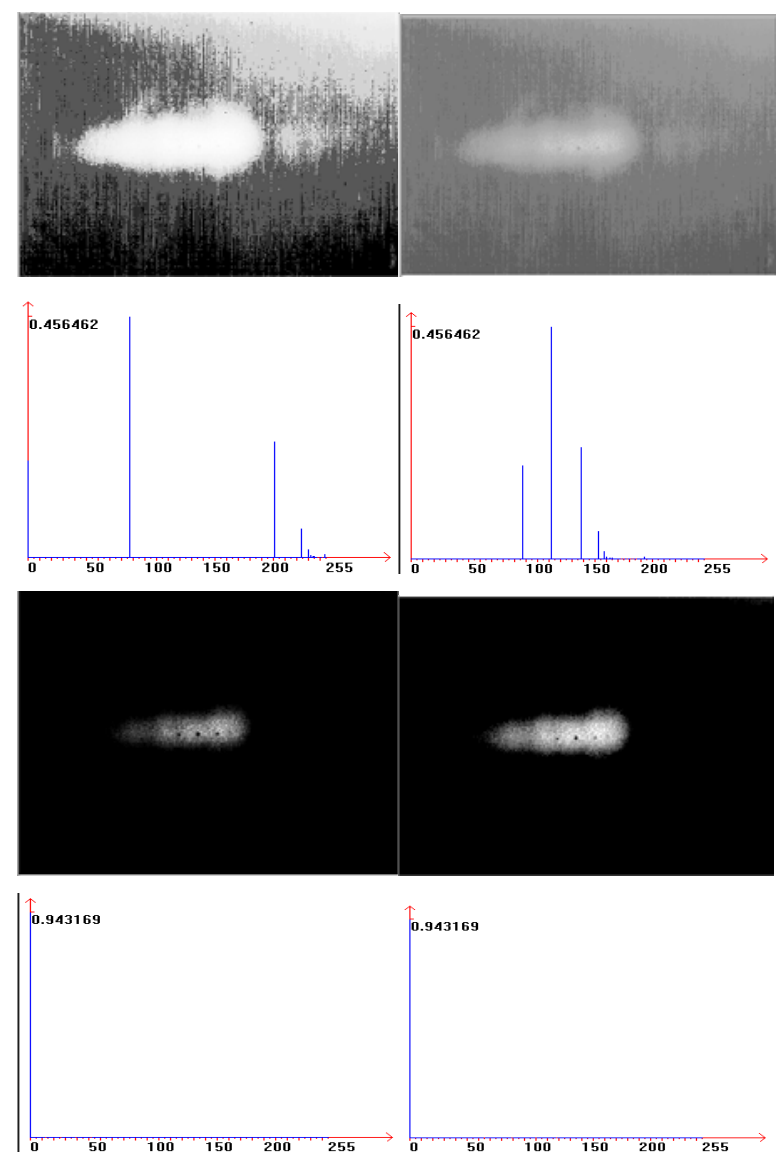

Figure 4. a) Histogram Equalization of Far Infrared Laser Image with its histogram b)Double Plateaus Histogram Equalization of Far Infrared Laser Image with its histogram (Upper Threshold=0.1, Lower Threshold=0.01) Self-adaptive Enhancement of Far Infrared Laser Image with its histogram ( $11=0, y 2=240, \mathrm{n}=3, \mathrm{X} 1=33 \mathrm{x} 2=53$ ).

TABLE I. COMPARISON OF RESULTS FROM DIFFERENT ENHANCEMENT ALGORITHMS FOR NEAR INFRARED LASER IMAGE ( $\beta=10000)$

\begin{tabular}{|c|c|c|c|c|c|}
\hline & $C_{\text {contrast }}$ & SNR & $C_{\text {total }}$ & MSE \\
\hline \multicolumn{2}{|c|}{ Hist. Equ. } & 5566.908203 & -12.35614 & -6.910486 & 761.914490 \\
\hline \multicolumn{2}{|c|}{ Doubl Plateaus } & 981.169983 & -0.893734 & -0.08682 & 213.979782 \\
\hline \multirow{6}{*}{ 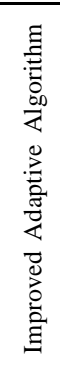 } & $\begin{array}{l}\mathrm{X} 1=31 \\
\mathrm{X} 2=137\end{array}$ & 1281.437134 & 1.541823 & 0.197575 & 150.934814 \\
\hline & $\begin{array}{l}\mathrm{X} 1=31 \\
\mathrm{X} 2=153\end{array}$ & 707.042908 & 2.894436 & 0.204649 & 140.083328 \\
\hline & $\begin{array}{l}X 1=31 \\
X 2=200\end{array}$ & 552.949158 & 3.087594 & 0.170728 & 137.679443 \\
\hline & $\begin{array}{l}\mathrm{X} 1=31 \\
\mathrm{X} 2=137\end{array}$ & 1491.130371 & 1.147759 & 0.171146 & 155.931030 \\
\hline & $\begin{array}{l}X 1=31 \\
X 2=153\end{array}$ & 1464.628662 & 1.221669 & 0.178929 & 155.553772 \\
\hline & $\begin{array}{l}X 1=31 \\
X 2=200\end{array}$ & 1575.262451 & 0.957959 & 0.150904 & 157.975037 \\
\hline
\end{tabular}


TABLE II. COMPARISON OF RESULTS FROM DIFFERENT ENHANCEMENT ALGORITHMS FOR FAR INFRARED LASER IMAGE

\begin{tabular}{|l|c|c|c|c|}
\hline \multicolumn{5}{|c|}{$(\beta=10000)$} \\
\hline & $C_{\text {canurt }}$ & SNR & $C_{\text {total }}$ & MSE \\
\hline $\begin{array}{l}\text { Hist. Equ. } \\
\text { Poubl }\end{array}$ & 4276.423340 & -15.268683 & -6.529535 & 230.337082 \\
\hline Adapteaus & 848.740295 & -15.585990 & -1.322846 & 258.170044 \\
\hline $\begin{array}{l}\text { Improved } \\
\text { Algorithm }\end{array}$ & 474.962921 & -1.340687 & -0.06367 & 45.223053 \\
\hline
\end{tabular}

TABLE III. COMPARISON OF RESULTS FROM DIFFERENT ENHANCEMENT ALGORITHMS FOR FAR INFRARED LASER IMAGE $(\beta=10000)$

\begin{tabular}{|l|c|c|c|c|}
\hline & $C_{\text {courum }}$ & SNR & $C_{\text {total }}$ & MSE \\
\hline Hist. Equ. & 4081.262695 & -13.98088 & -5.705967 & 230.83625 \\
\hline Doubl Plateaus & 569.48883 & -10.71792 & -0.61590 & 170.34597 \\
\hline Adaptive & 492.9458 & -0.705361 & -0.03477 & 51.698330 \\
\hline $\begin{array}{l}\text { Improved } \\
\text { Algorithm }\end{array}$ & 1306.844849 & -2.581531 & -0.33736 & 56.488583 \\
\hline
\end{tabular}

4. When applying the self-adaptive algorithm, thresholds $x_{1}=30$ and $x_{2}=135$ are get through the calculation of taking $3 \times 3$ cross window. In this algorithm, the peak in the histogram of original image is suppressed almost all to close the 0 grey level, the grey level interval of uninterested areas is suppressed almost all to close to the 255 grey level, and the grey level interval of the targets is extend to almost all grey level interval from 0 to 255. Hence, the targets are greatly enhanced.

5. However, when the points by human option are different in adaptive linear transformation, the effect of the segmentation are different. It can be analyzed that the basic reason if the randomness of $\mathrm{x} 2$ from the perspective of comparative data. But when we take the local histogram equalization on the results of linear transformation, the processing affection get from the three situations eventually are almost the same. It is worthwhile to note that from the perspective of data comparison, with the same X1,X2, $C_{\text {total }}$ and MSE get from the algorithm in this paper is much poor in theory than that from the adaptive piecewise linear transformation. But, algorithm in this paper is a little better than the adaptive piecewise linear transformation on the stability of the processing affection and the overall enhancement effect, of which the main reason is that:

In the adaptive piecewise linear transformation, the quality of the image is decided by the size of X2 directly and we could get the light and shade degree of the enhanced image, and $C_{\text {contrast }}$ get smaller, image get shaded when x2 get larger. This is because that when x2 is getting larger, much less uninterested gray level are compressed into 255 and the image looks darker. But if we add the local improved histogram equalization to the linear transformation, $C_{\text {contrast }}$ is improved obviously, and the overall effect of the target is greatly improved too.

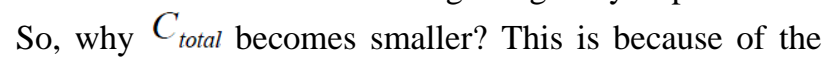

deciding factor SNR of $C_{\text {total }}$, with the same value of x1 and $\mathrm{x} 2$, the value of SNR get from the algorithm in this paper is much lower than that from adaptive segmentation linear transformation. In a sense, this algorithm takes the small range reduced for the sharp rise of $C_{\text {contrast }}$, which although makes $C_{\text {total }}$ lower, but from the perspective of sight, the effect the enhancement is greatly improved.

\section{CONCLUSION}

This paper presented a self-adaptive enhancement algorithm for infrared images. The histogram of the global image is divided into three parts: background area, objectives area, and uninterested area. The background and noise containing in the image will be restrained, and the targets will be highlighted. Experimental and quantitative evaluation results presented in this paper demonstrate that the algorithm performs well when compared with several approaches to image enhancement, such as histogram equalization, double plateaus histogram equalization and so on, which are available in the literature.

\section{REFERENCES}

[1] Zhang CJ, Wang XD, Zhang HR, et al.: An anti-noise algorithm for enhancing global and local contrast for infrared image, International Journal of Wavelets Multiresulution and Information Processing, Vol.5(1), 101112 (2007)

[2] Song, Y.F.; Shao, X.P.; Xu, J.; New enhancement algorithm for infrared image based on double plateaus histogram, Infrared and Laser Engineering (in Chinese), Vol.37(2), 2008:308-311

[3] Wallis, R.; An approach to the Space Variant Restoration and enhancement of images, Proc Symp on Current Mathematical Problems in Image Science, Navel Postgraduate School, Monterey, CA, 235-241(1976)

[4] Atutaleb, S. A.; Automatic thresholding of gray-level pictures using two-dimension entropy, Computer Vision, Graphics and Image Processing, 47(1): 22-32(1989)

[5] Highnam, R.; Brady, M.; Model-based image enhancement of far infrared images, IEEE Transactions on Pattern Analysis and Machine Intelligence, Vol.19(4), 410 - 415 (1997)

[6] Tang, M.; Ma, S.D.; and Xiao, J.; Model-based adaptive enhancement of far infrared image sequences, Pattern Recognition Letters, Vol.21(9), 827-835(2000)

[7] Gilboa, G.; Sochen, N.; Zeevi, Y. Y.; Forward-andbackward diffusion processes for adaptive image enhancement and denoising, IEEE Trans. Image Process., vol. 11, no. 7, 689-703(2002)

[8] Li, Y.H.; Wang, C.; Zhou, J.Z.; Liu, Q.M.; Real-time Infrared Laser Measurement System Design and Development, Proceedings of 2009 WRI World Congress on Software Engineering, Vol. 1, 435-439(2009)

[9] Rosenfield, A.; Kak, A.C.; Digital Picture Processing, New York, Academic Press, 1982.

[10] Zhang, C.J.; Yang, F.; Wang, X.D.; Zhang, H.R.;An Efficient Nonlinear Algorithm for Contrast Enhancement of Infrared Image, Proceedings of the Fourth International Conference on Machine Learning and Cybernetics, Guangzhou, 18-21 August 2005.

[11] Yeong-Taeg Kim, IEEE Transactions on Consumer Electronics, Vol. 43, No. 1, FEBRUARY 1997 